\title{
More Hemorrhagic and Severe Events Cause Higher Hospitalization Care Cost for Childhood Stroke in Taiwan
}

\author{
Pei-Chun Chen, MS, Kuo-Liong Chien, MD, PhD, Ching-Wen Chang, PhD, Ta-Chen Su, MD, PhD, \\ Jiann-Shing Jeng, MD, PhD, Yuan-Teh Lee, MD, PhD, and Fung-Chang Sung, PhD
}

Objective Rarely has childhood stroke been compared with adult stroke for incidence or cost. This population study compared the stroke incidence and the associated hospitalization care costs between children and adults in Taiwan.

Study design We used reimbursement claims data from the National Health Insurance program to identify stroke diagnoses in 1997 to 2003. The inpatient costs of both the first admission and recurrent stroke from 1979 childhood cases and 365,169 adult cases were compared by age and stroke subtype, excluding those less than 1 month of age.

Results The mean inpatient costs were higher for patients $<10$ and 10 to 19 years of age (\$3565 per case) compared with adult cases (\$1933), including both first and recurrent hospitalizations, and they were higher for the recurrent cases. Patients $<10$ years old had the highest proportional incidence of hemorrhage events $(\mathbf{7 1 . 4 \% )}$, followed by patients in the 10 - to 19-year-old group (61.4\%), and the lowest for adults (21.3\%). Hemorrhagic events incurred 2 to 12 times higher cost than other types of stroke.

Conclusions The hospitalization care costs for stroke are higher for children than for adults because of a greater proportion of hemorrhagic cases among children. (J Pediatr 2008;152:388-93)

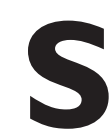

troke is a costly disease. The techniques used for diagnosis, medication used for lifelong care, and the long-term rehabilitation required for survivors are an enormous burden on healthcare expenditure. ${ }^{1-3}$ Although the mortality rate ${ }^{4}$ and case fatalities ${ }^{5,6}$ from stroke have declined over recent decades, it is still among the top 3 causes of death in most developing countries. ${ }^{4,7}$ There is uncertainty whether the mortality rate reduction is due to a decrease in incidence, improvement in survival, or both. Several studies have suggested that the incidence of stroke may have reached a plateau or even increased in some areas ${ }^{8,9}$ and that the hospitalization rate is increasing as well. ${ }^{10}$ This indicates that the burden on healthcare for stroke remains high.

Many studies estimated hospital care ${ }^{11-14}$ and lifetime ${ }^{15}$ costs for stroke. The cost varies with the stroke subtype. ${ }^{11,12,15}$ The severity of the disease, length of stay (LOS) at hospital, and sequelae after discharge are important factors for the variation in cost. ${ }^{3,11,13}$ Reed et $\mathrm{al}^{12}$ reported that patients with subarachnoid hemorrhage (SAH) incurred the highest costs, followed by intracerebral hemorrhage (ICH), ischemic cerebral infarction, and transient cerebral ischemia (TIA). However, this study focused on adults rather than the young.

Estimates of the incidence rates for childhood stroke ranged from 2.1 to 13 per 100,000 children-years in Hong-Kong, ${ }^{16}$ the United States, ${ }^{17}$ and France. ${ }^{18}$ Although they are uncommon, children who have strokes may have long-term morbidity and economically adverse neurologic consequences. ${ }^{19}$

In Taiwan, the mortality rate from stroke is high, ranking the second-most common cause of death. ${ }^{20}$ There are limited studies on the occurrence of childhood stroke in Taiwan and no studies on the medical costs of stroke in children. The goals of this study were to compare the occurrence of stroke by subtype and the associated cost of hospitalization between children and adults.

\begin{tabular}{llll}
\hline HS & Hemorrhage stroke & LOS & Length of stay \\
ICD-9-CM & International classification of disease-9-clinical & NHI & National health insurance \\
& modification & OHH & Other intracranial hemorrhage \\
ICH & Intracerebral hemorrhage & SAH & Subarachnoid hemorrhage \\
IS & Ischemic stroke & TIA & Transient cerebral ischemia \\
\hline
\end{tabular}

From the Institution of Environmental Health (P.C., C.C., F.S.), the Institution of Preventive Medicine (K.C., Y.L., F.S.), the Department of Internal Medicine (K.C., T.S., Y.L.), and the Department of Neurology (J.).), National Taiwan University Hospital, Taipei, and the Institution of Environmental Health (F.S.), China Medical University College of Public Health, Taichung, Taiwan.

Supported by the National Science Council, Executive Yuan, Taiwan, Grant NSC9423।4-B-039-006, 2005-2006.

Submitted for publication Jan 31, 2007; last revision received Jun 19, 2007; accepted Aug 3, 2007.

Reprint requests: Fung-Chang Sung, PhD, $\mathrm{MPH}$, Institute of Environmental Health, China Medical University College of Public Health, 9I Hsueh-Shih Rd, Taichung 404, Taiwan. E-mail: fcsung@mail.cmu.edu.tw; sung@ha.mc.ntu.edu.tw.

0022-3476/\$ - see front matter

Copyright (C) 2008 Mosby Inc. All rights reserved.

10.10 | 6/j.jpeds.2007.08.003 


\section{METHODS}

\section{Database and Study Population}

The National Health Insurance (NHI), a universal health program, was established in Taiwan in 1995. The NHI covers more than $96 \%$ of the population and has contracted with $97 \%$ of the hospitals in Taiwan. ${ }^{21,22}$ Its medical claims data for reimbursement have been computerized since 1996 . Hospitalization data were obtained for this study from the National Health Research Institute, which maintains the NHI database. The data contained information on sex, birthday, and patient encrypted identification numbers, dates of admission and discharge, function types, LOS as inpatients, discharge diagnoses (up to 5), operation procedures (up to 5), discharge disposition and costs (i.e. expenditures) by admission. The cost components include fees for diagnosis, room and meals, laboratory tests, radiography services, therapeutic treatment, operation, rehabilitation, blood products, hemodialysis, anesthetic, special medical supply, medication, and other services. All first admissions for stroke from 1997 to 2003, excluding neonates (age less than 1 month), formed the study population. Their succeeding admissions were also identified for the cost analysis. Inpatients with stroke were identified using primary discharge diagnosis for ICD-9-CM code 430 to 437 . Three inpatients with a total cost 100 times greater than the average cost were excluded from the cost analyses.

\section{Definitions of Stroke Subtypes, Costs and Length of Stay at Hospital}

The stroke types were classified according to the following ICD-9-CM codes: 430, subarachnoid hemorrhage (SAH); 431, intracerebral hemorrhage ( $\mathrm{ICH}) ; 432$, other intracranial hemorrhage (OIH); ischemic stroke (IS), including 433 (occlusion and stenosis of precerebral arteries), 434 (occlusion of cerebral arteries); 435, transient cerebral ischemia (TIA); and other cerebrovascular disease (others), including 436 (acute, but ill-defined cerebrovascular disease) and 437 (other and ill-defined cerebrovascular disease). For each admission and discharge, the total medical costs were estimated by use of the sum of all cost components. The duration between admission and discharge dates were calculated as LOS. Costs expressed in this study were in US dollars (\$1 U.S. is approximately $\$ 33$ in Taiwanese currency).

\section{Statistical Analysis}

We plotted mean costs and LOS of the first admission and succeeding hospitalizations of recurrent stroke to compare the trends by age. We also estimated age-, sex-, and subtype-specific stroke incidence rates with the first stroke admissions divided by the population person-years for the study in the age bands $<10,10$ to 19 , and $\geq 20$ years. $^{23}$ Further analysis compared average and median costs between young children, adolescents, and adults by stroke subtype for the first and succeeding admissions. One-way analysis of
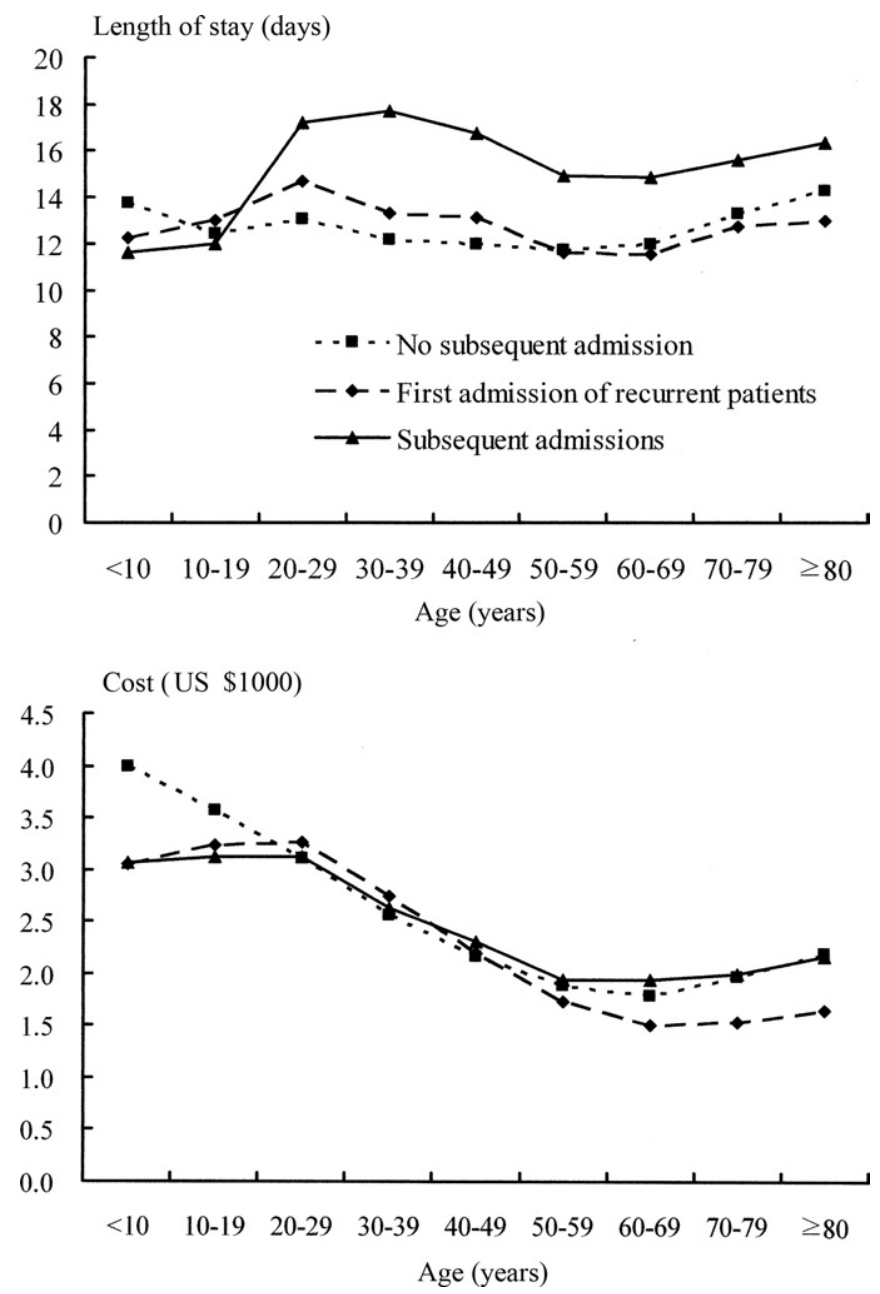

Figure. Length of stay and average cost during hospitalization care for stroke by patient's age for first and recurrent admissions in Taiwan, 1997-2003.

variance was used to examine the differences in mean costs among these 3 age groups. We tested for the mean cost trends across age groups with the linear trend test (GLM procedure). Two multiple linear regression models were used to assess the excess cost associated with age controlling for sex, stroke subtypes, LOS, type of hospital services, outcome at discharge (dead or not), and seasonal and secular factors. In the second model, we compared costs between the first hospitalizations and subsequent hospitalizations for patients with recurrent events. Further regression analysis was also performed with natural-log transformation for costs and LOS in the regression equation. The linear regression assumptions, potential outliers and influential points in the model were also examined. The statistical significance level was set at $P<.05$. All analyses were performed with SAS 9.1 (SAS Institute Inc., Cary, NC).

\section{RESULTS}

For the period 1997 to 2003 , we identified 209,904 (57.2\%) men (and boys) and 157,244 (42.8\%) women (and girls) as stroke incidence cases from the initial hospitalization 
Table I. Annual average stroke incidence rate for each subtype by age and sex, Taiwan, 1997-2003

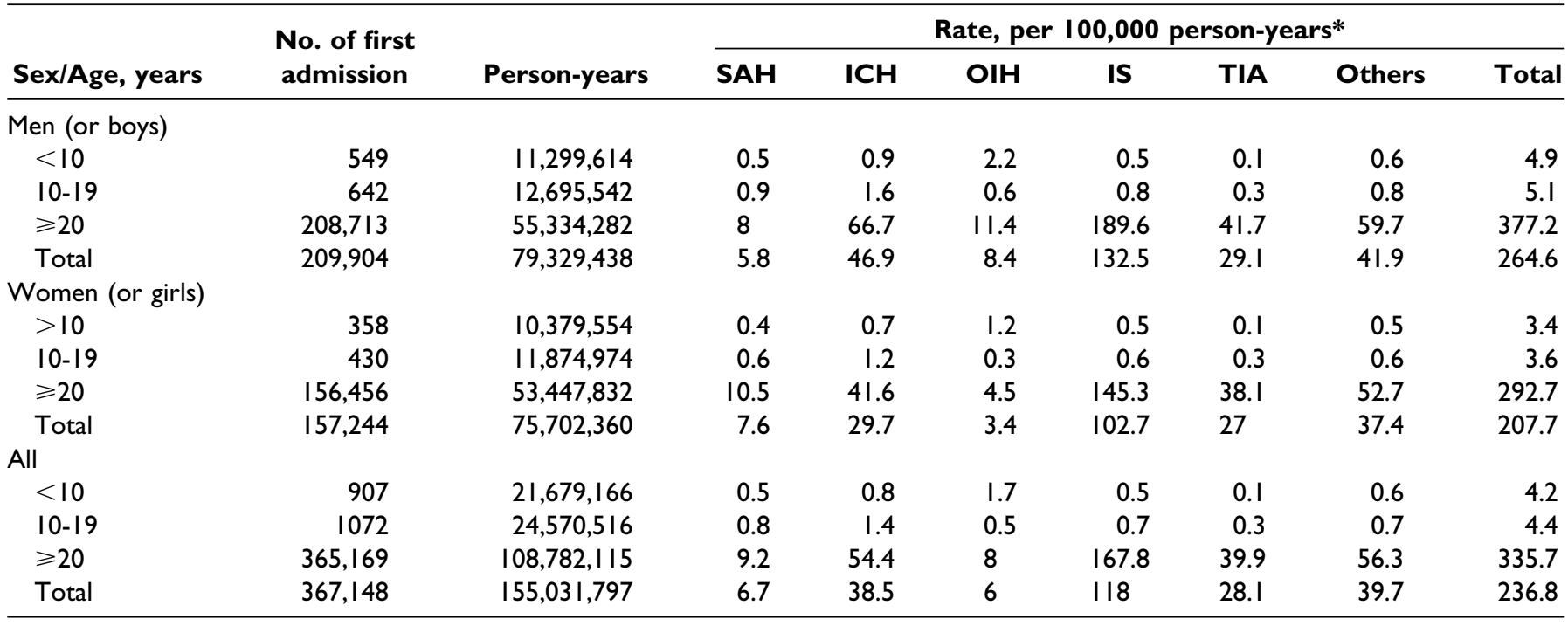

*Numbers of first admission for stroke per 100,000 person-years in each subgroup during the study period.

records. Among children (1 month to 19 years of age), the average stroke incidence rate was highest in those $<5$ years old (6.1 per 100,000), followed by $15-$ to $19-, 10-$ to $14-$, and 5- to 9-year-old children, and was higher in boys than in girls (data not shown). The average cost of care during the first hospitalization declined with increasing age to the lowest for 60- to 69-year-old patients (Figure). The average LOS ranged approximately 11 to 13 days among age groups with no recurrent admission, except for the youngest and the oldest who exhibited an upward trend. The average LOS for subsequent admissions was 2.3 to 4.5 days greater in adults. For patients with no subsequent admissions, the highest mean cost was $\$ 3991$ for $<10$-year-old children, and the lowest mean cost was $\$ 1780$ for 60 - to 69 -year-old patients. The average costs and LOS of succeeding hospitalizations for recurrent cases were higher than those for the initial hospitalization among adults but not children. The overall average daily cost decreased from approximately US $\$ 279$ for children $<10$ years old to $\$ 129$ for patients 70 to 79 years old (data not shown).

Table I shows that about half (49.8\%) of patients were hospitalized for ischemic events (118 per 100,000 personyears), and $21.6 \%$ of patients had hemorrhage-related strokes (SAH, ICH, and $\mathrm{OIH}$ ) (51.2 per 100,000 person-years). Approximately $60 \%$ to $70 \%$ of strokes among children were due to hemorrhage, and IS was the major type among adult patients (50\%). The relative incidence rates of hemorrhage events were higher in patients $<10$ years old $(71.4 \%)$ and 10 to 19 years old $(61.4 \%)$ than in adults $(21.3 \%)$. The stroke distributions by subtype among these age groups were approximately similar for both sexes.

Table II (available at www.jpeds.com) shows the average and median costs for patients with stroke by stroke subtype and age. Overall, the average costs were higher for recurrent events than for the initial events. Inpatients with hemorrhagic events had higher average costs than those with ischemic and other events. Among the initial hospitalization, SAH was the most costly stroke subtype on average (\$6098), followed by ICH, OIH, IS and other type, with TIA the least expensive (\$501). The mean costs were higher in children than in adults among inpatients with ICH, IS, and other types of stroke $(P<.001)$. The mean costs had significant inverse linear relationships with age except for inpatients with SAH and TIA. These patterns remained when the cost associations with age and stroke subtype were evaluated with median costs. For patients with SAH, the mean cost was higher in adults than in other age groups $(P=.001)$.

In the multiple regression analysis, type of hospital, sex, age, stroke subtype, LOS, outcome at discharge, and seasonal/secular factors were significant predictors of the hospital care cost for stroke and explained $60.1 \%$ of the variation for patients with no subsequent admission (Model 1 in Table III). Compared with adults, excess costs for inpatient care were $\$ 765.4$ for $<10$-year-old children and $\$ 236.9$ for 10 - to 19 year-old adolescents $(P<.001$ for both). Overall, the cost for SAH was notably higher than the cost for other types of stroke, and the difference between $\mathrm{ICH}$ and $\mathrm{OIH}$ was negligible. A similar pattern was found when the regression analysis included only recurrent cases (Model 2).

\section{DISCUSSION}

This study revealed an important pattern by highlighting the relationship between childhood stroke subtype and cost, which has not been reported in previous studies. Taiwan's NHI is a single-payer, government-run, mandatoryenrollment health insurance program, which aims to ensure equitable access to health care for all ages and to provide a wide scope of health care services. ${ }^{24}$ This insurance scheme enabled us to obtain the representative cost estimations by stroke subtype for all ages from the nationwide hospitalization database. 


\begin{tabular}{|c|c|c|c|c|}
\hline & Mode & & Model & \\
\hline & Coefficient (SE) & $\mathbf{P}$ & Coefficient (SE) & $\boldsymbol{P}$ \\
\hline Intercept & $-608.4(19)$ & $<.001$ & $-663.3(22.7)$ & $<.001$ \\
\hline Hospital level (vs. Community Hospital & & & & \\
\hline Medical center & $424.8(12.8)$ & $<.001$ & $813(13.3)$ & $<.001$ \\
\hline Regional hospital & $262(11.7)$ & $<.001$ & $359.8(11.9)$ & $<.001$ \\
\hline Sex, men or boys (vs women or girls) & $-54.9(9.5)$ & .005 & $-57.1(10.2)$ & $<.001$ \\
\hline Age, years (vs $\geq 20$ ) & & & & \\
\hline$<10$ & $765.4(92.3)$ & $<.001$ & $562.6(\mid 25.2)$ & $<.001$ \\
\hline $10-19$ & $236.9(83.7)$ & $<.001$ & $314.2(125.4)$ & .012 \\
\hline Stroke subtype (vs TIA) & & & & \\
\hline $\mathrm{SAH}$ & $3693(31.5)$ & $<.001$ & $3430.4(39.3)$ & $<.001$ \\
\hline $\mathrm{ICH}$ & I I66.1 (18.3) & $<.001$ & $1238.9(21.4)$ & $<.001$ \\
\hline $\mathrm{OlH}$ & $1060.2(31.9)$ & $<.001$ & $1061.2(40.4)$ & $<.001$ \\
\hline IS & $-34.3(14.9)$ & .022 & $110.8(17.7)$ & $<.001$ \\
\hline Others & $70.2(17.6)$ & $<.001$ & $110(19.9)$ & $<.001$ \\
\hline Length of stay, days & $136(0.2)$ & $<.001$ & $101.1(0.2)$ & $<.001$ \\
\hline Deceased at discharge (vs alive) & $1790.9(26.6)$ & $<.001$ & $2161.4(47.3)$ & $<.001$ \\
\hline Year of admission (vs 1997-1998) & & & & \\
\hline $1999-2000$ & $177.7(\mid 2.8)$ & $<.001$ & $193.8(13.5)$ & $<.001$ \\
\hline $2001-2003$ & $296.8(11.6)$ & $<.0001$ & $364.5(12.7)$ & $<.001$ \\
\hline Season of admission (vs spring) & & & & \\
\hline Summer & $10.3(13.4)$ & .442 & $15.8(14)$ & .261 \\
\hline Fall & $43.2(13.3)$ & .001 & $31.8(14.1)$ & .024 \\
\hline Winter & $42.6(13.2)$ & .001 & $31.4(14)$ & .026 \\
\hline First-hospitalized (vs recurrent) & - & - & $-130.5(10.6)$ & $<.001$ \\
\hline
\end{tabular}

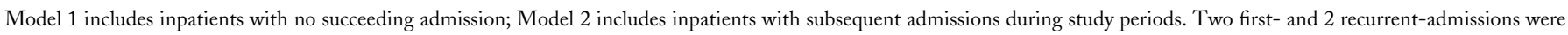
excluded because of extremely high cost.

*Adjusted $\mathrm{R}^{2}$ : 0.601 for model 1; 0.54 for model 2 .

The principal determinants in stroke hospitalization care cost are LOS, stroke subtype, and the severity of the disease. ${ }^{3}$ Our analysis indicated a downward trend in average hospitalization costs as patient's age increased, even though the LOS of the initial admission were similar among most age groups except for the elderly and the 20- to 29-year-old patients. The association between age and inpatient cost was controversial in previous studies, which focused on adulthood stroke. In the United States, after adjusting for covariates, inpatients aged 80 to 89 and $\geq 90$ years old are $30 \%$ and $53 \%$ less costly, respectively, compared with those 19 to 49 years of age, ${ }^{12}$ a trend somewhat similar to our study.

In Japan, the cost of inpatient care for IS and ICH in those less than 70 years of age is similar to that of older patients. ${ }^{14}$ Another earlier study in the U.S. suggested that demographic factors such as age and sex accounted for less than $10 \%$ of the variation in hospitalization cost, whereas LOS explained more than $72 \%$ of the variation. ${ }^{11}$ Our analysis suggests that the age of patients and stroke types have a greater implication for cost than LOS has, especially when considering childhood stroke. The highest average cost occurs on those $<10$ years of age for the initial hospitalization, followed by 10 - to 19 -year-old patients, and independent of the stroke subtype, LOS, sex, and survival status at discharge. However, the cost was the highest for 20- to 29-year-old patients in the recurrent cases. Further analysis showed that hemorrhagic stroke also had greater cost in these recurrent cases.

We confirmed the observation of previous studies that IS (or cerebral infarction) is much more common than hemorrhagic events in adulthood stroke. ${ }^{25-27}$ For childhood stroke, several population studies have shown variation among geographic areas. ${ }^{16-18,28-31}$ The first study conducted in the United States reviewed medical records from a Rochester, Minnesota, records-linkage system from 1965 to1974 for 15,834 children $<15$ years old of age. ${ }^{28}$ The incidence rate of HS was higher than that of IS (1.89 vs 0.63 per 100,000). Two other studies with short observation period, also for children of $<15$ years in Cincinnati $(n=295,577)$ and Washington, DC ( $\mathrm{n}=773,016)$, revealed less remarkable incidence differences between IS and HS. ${ }^{29,30}$ A study in California $(\mathrm{n}=9,907,432)$ reported incidence rates of 2.4 / 100,000 for all strokes, and 1.2/100,000 for both IS and HS. ${ }^{17}$ A recent study in Texas $(n=92,418)$ yields much greater incidence for HS than for IS (3.2 vs 1.1 per 100,000). ${ }^{31}$ However, the U.S. National Hospital Discharge Survey from 1980 to 1998 showed that, among all stroke events, the rate of hospitalization for IS was higher than that for SAH and ICH together among children aged 0 to 18 years old ( 7.8 vs 2.9 per 100,000 children per year). ${ }^{32}$ Higher incidence rates for IS than for HS have also been reported in the Hong-Kong childhood stroke registry ${ }^{16}$ and in Dijon, 
France. ${ }^{18}$ No cost analysis for the care of childhood stroke is available in the above studies.

Children in Taiwan are more likely than children in other areas to have HS, a notable difference from adults. Although Asians are at higher risk than Caucasians for $\mathrm{ICH}$ and $\mathrm{SAH},{ }^{33}$ the different pattern we found is not explained well by genetic variation because children in both HongKong $^{16}$ and Taiwan are predominantly Chinese. A previous study demonstrated a striking variance in stroke subtypes among adult populations in different provinces of China. ${ }^{26}$ Although all the populations are from the same race, the percentages of HS varied from $19 \%$ to $42 \%$ of strokes. Furthermore, compared with non-Hispanic white, Asians/Pacific Islanders in the United States have a greater geographic variation of stroke incidence, indicating a significant interaction between race and regional variation. ${ }^{33}$

This study confirmed that it costs more to care for hemorrhagic events than for ischemic events, consistent with earlier studies. ${ }^{11,12,14} \mathrm{SAH}, \mathrm{ICH}$, and $\mathrm{OIH}$ cost the most among all stroke events, and these types of stroke are at higher proportional incidence in children in Taiwan. The high cost among children, found in this study, is well explained by the high HS incidence. The disease may be more severe and complicated in children than in adults, requiring more intensive care and complex treatments or procedures, resulting in high cost.

The increased cost for the children may be related to physiological and psychological elements. The frequently encountered causes of pediatric stroke, different from that for adults, may include heart disease, moyamoya disease, hereditary diseases, and vascular anomalies. ${ }^{16,32}$ These diseases require more investigation such as angiography, echocardiography, coagulation, and hematologic studies. More specific therapies such as endovascular therapy may be performed for stroke in children. Also, parents' expectation for stroke care in children may be higher. Further analysis of our data showed that childhood stroke cases did undergo more radioactive scans and therapeutic treatment than adults. They also undergo more surgeries, particularly for ICH ( $46.6 \%$ for patients $<10$ years old, $44.1 \%$ for patients 10 to 19 years old, and $28.9 \%$ for all adults in average). The average fees of surgery, laboratory, and medicine were notably higher for children with this type of stroke (data not shown).

There are limitations in this study. First, our study excluded uninsured patients with stroke. However, with an insurance coverage rate of $96 \%$, our estimation is believed to be representative for the population of Taiwan. Second, this study did not include outpatient care and non-hospital costs. It is important to include these costs for evaluating the overall economic impact of stroke, although our study aimed to observe the pattern of stroke admission. Third, the diagnostic practice by ICD code may not be totally the same in different areas. But it is unlikely to alter the pattern of stroke subtype among age groups we observed because the use of neurologic imaging techniques is now widespread. Finally, the multiple regression analysis might be affected by the skewed distribu- tions in cost and LOS, and the skewed LOS distribution among patients with different stroke subtypes might affect the cost estimation. However, our focus was the comparison of hospital care cost among children, adolescents, and adults, and the inverse association between these age groups and cost was robust after controlling for these covariates. The cost associated with age and the subtype of stroke was similar in pattern whether the cost is estimated for whole hospital care or cost per day.

Findings from this study suggest a higher inpatient cost for childhood stroke in Taiwan than adulthood stroke. The disparity in cost is associated with the pattern of strokesubtype and the complexity of the disease.

\section{REFERENCES}

1. Taylor TN, Davis PH, Torner JC, Holmes J, Meyer JW, Jacobson MF. Lifetime cost of stroke in the United States. Stroke 1996;27:1459-66.

2. Caro JJ, Huybrechts KF, Duchesne I. Management patterns and costs of acute ischemic stroke: an international study. For the Stroke Economic Analysis Group. Stroke 2000;31:582-90.

3. Martinez-Vila E, Irimia P. The cost of stroke. Cerebrovasc Dis 2004;17 (Suppl 1):124-29.

4. Feigin VL, Lawes CM, Bennett DA, Anderson CS. Stroke epidemiology: a review of population-based studies of incidence, prevalence, and case-fatality in the late 20th century. Lancet Neurol 2003;2:43-53.

5. Numminen H, Kotila M, Waltimo O, Aho K, Kaste M. Declining incidence and mortality rates of stroke in Finland from 1972 to 1991. Results of three populationbased stroke registers. Stroke 1996;27:1487-91.

6. Morikawa Y, Nakagawa H, Naruse Y, Nishijo M, Miura K, Tabata M, et al. Trends in stroke incidence and acute case fatality in a Japanese rural area: the Oyabe study. Stroke 2000;31:1583-7.

7. Sarti C, Rastenyte D, Cepaitis Z, Tuomilehto J. International trends in mortality from stroke, 1968 to 1994. Stroke 2000;31:1588-1601.

8. Bonita R, Broad JB, Beaglehole R. Changes in stroke incidence and case-fatality in Auckland, New Zealand, 1981-91. Lancet 1993;342:1470-3.

9. Feigin VL, Wiebers DO, Whisnant JP, O'Fallon WM. Stroke incidence and 30-day case-fatality rates in Novosibirsk, Russia, 1982 through 1992. Stroke 1995; 26:924-9.

10. Fang J, Alderman MH. Trend of stroke hospitalization, United States, 19881997. Stroke 2001;32:2221-6.

11. Holloway RG, Witter DM Jr, Lawton KB, Lipscomb J, Samsa G. Inpatient costs of specific cerebrovascular events at five academic medical centers. Neurology 1996;46:854-60.

12. Reed SD, Blough DK, Meyer K, Jarvik JG. Inpatient costs, length of stay, and mortality for cerebrovascular events in community hospitals. Neurology 2001;57:305-14. 13. Chang KC, Tseng MC. Costs of acute care of first-ever ischemic stroke in Taiwan. Stroke 2003;34:e219-21.

14. Yoneda Y, Okuda S, Hamada R, Toyota A, Gotoh J, Watanabe M, et al. Hospital cost of ischemic stroke and intracerebral hemorrhage in Japanese stroke centers. Health Policy 2005;73:202-11.

15. Dewey HM, Thrift AG, Mihalopoulos C, Carter R, Macdonell RA, McNeil JJ, et al. Lifetime cost of stroke subtypes in Australia: findings from the North East Melbourne Stroke Incidence Study (NEMESIS). Stroke 2003;34:2502-7.

16. Chung B, Wong V. Pediatric stroke among Hong Kong Chinese subjects. Pediatrics 2004;114:e206-12.

17. Fullerton HJ, Wu YW, Zhao S, Johnston SC. Risk of stroke in children: ethnic and gender disparities. Neurology 2003;61:189-94.

18. Giroud M, Lemesle M, Gouyon JB, Nivelon JL, Milan C, Dumas R. Cerebrovascular disease in children under 16 years of age in the city of Dijon, France: a study of incidence and clinical features from 1985 to 1993. J Clin Epidemiol 1995;48:1343-8. 19. Lanthier S, Carmant L, David M, Larbrisseau A, de Veber G. Stroke in children: the coexistence of multiple risk factors predicts poor outcome. Neurology 2000; 54:371-8.

20. Summary of cause of death statistics, Taiwan, 2000-2004. Available at www. doh.gov.tw/statistic/index.htm. Accessed January 16, 2007.

21. Lu JF, Hsiao WC. Does universal health insurance make health care unaffordable? Lessons from Taiwan. Health Aff 2003;22:77-88.

22. Chiang TL. Taiwan's 1995 health care reform. Health Policy 1997;39:225-39. 
23. Health and vital statistics, Taiwan, 1997-2003. Available at www.doh.gov.tw/ statistic/index.htm. Accessed January 16, 2007.

24. Cheng TM. Taiwan's new national health program: genesis and experience so far. Health Aff 2003;22:61-76.

25. Hu HH, Sheng WY, Chu FL, Lan CF, Chiang BN. Incidence of stroke in Taiwan. Stroke 1992;23:1237-41.

26. Zhang LF, Yang J, Hong Z, Yuan GG, Zhou BF, Zhao LC, et al. Collaborative Group of China Multicenter Study of Cardiovascular Epidemiology: Proportion of different subtypes of stroke in China. Stroke 2003;34:2091-6.

27. Jerntorp P, Berglund G. Stroke registry in Malmo, Sweden. Stroke 1992; 23:357-61.

28. Schoenberg BS, Mellinger JF, Schoenberg DG. Cerebrovascular disease in infants and children: a study of incidence, clinical features, and survival. Neurology 1978; 28:763-8.
29. Broderick J, Talbot GT, Prenger E, Leach A, Brott T. Stroke in children within a major metropolitan area: the surprising importance of intracerebral hemorrhage. J Child Neurol 1993;8:250-5.

30. Earley CJ, Kittner SJ, Feeser BR, Gardner J, Epstein A, Wozniak MA, et al Stroke in children and sickle-cell disease: Baltimore-Washington Cooperative Young Stroke Study. Neurology 1998;51:169-76.

31. Zahuranec DB, Brown DL, Lisabeth LD, Morgenstern LB. Is it time for a large, collaborative study of pediatric stroke? Stroke 2005;36:1825-9.

32. Lynch JK, Hirtz DG, DeVeber G, Nelson KB. Report of the National Institute of Neurological Disorders and Stroke workshop on perinatal and childhood stroke. Pediatrics 2002;109:116-23.

33. Nguyen-Huynh MN, Johnston SC. Regional variation in hospitalization for stroke among Asians/Pacific Islanders in the United States: a nationwide retrospective cohort study. BMC Neurol Doi: 10.1186/1471-2377-5-21. 
Table II. Average hospital care cost (US\$) for first-hospitalized and recurrent strokes* by subtype and age, Taiwan, 1997-2003

\begin{tabular}{|c|c|c|c|c|c|c|c|c|c|c|c|c|c|c|}
\hline \multirow[b]{3}{*}{ Stroke subtype } & \multicolumn{12}{|c|}{ Age, years } & \multirow{3}{*}{$\begin{array}{c}P \\
\text { value } \dagger\end{array}$} & \multirow{3}{*}{$\begin{array}{c}P \\
\text { value } \neq\end{array}$} \\
\hline & \multicolumn{3}{|c|}{$<10$} & \multicolumn{3}{|c|}{$10-19$} & \multicolumn{3}{|c|}{$\geqslant 20$} & \multicolumn{3}{|c|}{ Total } & & \\
\hline & No. & Mean & Median & No. & Mean & Median & No. & Mean & Median & No. & Mean & Median & & \\
\hline \multicolumn{15}{|l|}{ SAH } \\
\hline First-hospitalized & 98 & 5531 & 3090 & 196 & 4120 & 1860 & 10,036 & 6142 & 3201 & 10,330 & 6098 & 3169 & .001 & .007 \\
\hline Recurrent & 9 & 2550 & 1715 & 32 & 6937 & 3064 & 2624 & 6335 & 3900 & 2665 & 6330 & 3863 & .297 & .435 \\
\hline \multicolumn{15}{|l|}{$\mathrm{ICH}$} \\
\hline First-hospitalized & 184 & 5255 & 3284 & 345 & 5125 & 2570 & 59,157 & 3912 & 1788 & 59,686 & 3923 & 1795 & $<.001$ & $<.001$ \\
\hline Recurrent & 34 & 4441 & 2830 & 58 & 2956 & $|84|$ & $|9,40|$ & 3894 & 1830 & 19,493 & 3892 & 1833 & .460 & .846 \\
\hline \multicolumn{15}{|c|}{$\mathrm{OlH}$} \\
\hline First-hospitalized & 374 & 3658 & 2167 & 116 & 3727 & 1579 & 8743 & 3104 & 1603 & 9233 & 3134 & 1621 & .108 & .044 \\
\hline Recurrent & 75 & 3157 & 1832 & 13 & 1875 & 1492 & 2624 & 3392 & 1683 & 2712 & 3378 & 1685 & .656 & .631 \\
\hline \multicolumn{15}{|l|}{ IS } \\
\hline First-hospitalized & 115 & 2442 & 1074 & 178 & 2478 & 1136 & 182,580 & 1554 & 704 & 182,873 & 1555 & 704 & $<.001$ & $<.001$ \\
\hline Recurrent & II & 1176 & 745 & 36 & 1466 & 1033 & 73,577 & 1845 & 812 & 73,624 & 1844 & 812 & .717 & .416 \\
\hline \multicolumn{15}{|l|}{ TIA } \\
\hline First-hospitalized & 13 & 764 & 323 & 68 & 485 & 349 & 43,442 & 501 & 370 & 43,523 & 501 & 369 & .549 & .547 \\
\hline Recurrent & 2 & 672 & 672 & 6 & 624 & 371 & 12,757 & 551 & 377 & 12,765 & 551 & 377 & .963 & .784 \\
\hline \multicolumn{15}{|l|}{ Others } \\
\hline First-hospitalized & 123 & 2540 & 1039 & 169 & |73| & 758 & 61,209 & 1094 & 462 & 61,501 & 1099 & 463 & $<.001$ & $<.001$ \\
\hline Recurrent & 86 & 2805 & 1846 & 60 & 2744 & |77| & 26,492 & 1323 & 552 & 26,638 & 1331 & 555 & $<.001$ & $<.001$ \\
\hline \multicolumn{15}{|l|}{ Total } \\
\hline First-hospitalized & 907 & 3837 & 1921 & 1072 & 3521 & |34| & 365,167 & 1897 & 676 & 367,146 & 1906 & 678 & $<.001$ & $<.001$ \\
\hline Recurrent & 217 & 3070 & 1826 & 205 & 3117 & $167 \mid$ & 137,475 & 2029 & 768 & 137,897 & 2032 & 770 & $<.001$ & $<.001$ \\
\hline
\end{tabular}

*Two first- and 2 recurrent-admissions were excluded because of extremely high cost.

$\dagger P$ values for differences among means were estimated with 1-way analysis of variance.

$\ddagger$ The trend of mean cost across ages was examined with linear trend testing. 